\title{
Insecticide resistance monitoring and evaluation in disease transmitting mosquitoes
}

\author{
Michael Coleman ${ }^{\dagger, \dagger \dagger}$ and Janet HemingWaY ${ }^{\dagger \dagger}, *$ \\ ${ }^{\dagger}$ Malaria Research Programme, Medical Research Council, Ridge Road, Durban, South Africa \\ ${ }^{\dagger \dagger}$ Liverpool School of Tropical Medicine, Pembroke Place, Liverpool, L3 5QA, United Kingdom
}

(Received October 6, 2006; Accepted January 16, 2007)

\begin{abstract}
The emergence of mosquito species resistant to insecticides widely used in malaria and dengue control has the potential to impact severely on the control of these disease vectors. A limited number of resistance mechanisms, including modification of the insecticides' target site, or changes in rates of metabolism involving esterases, glutathione S-transferases or monooxygenases operate in all insects. The potential for resistance to develop in vectors has been apparent since the 1950's, but the scale of the problem has been poorly documented. Few new public health insecticides have been developed for control of disease vectors for the past three decades and without good stewardship these insecticides will cease to be effective for vector control. This may have a dramatic effect in disease endemic countries, as few affordable alternative insecticides can rapidly be made available for vector control. Here we review our ability to optimally measure and manage insecticide resistance in field populations of insects, which is crucial to the long term sustainability of insecticide-based disease control campaigns. (C) Pesticide Science Society of Japan
\end{abstract}

Keywords: insecticide resistance, monitoring and evaluation, decision support systems.

\section{Introduction}

Mosquito borne diseases such as malaria and dengue cause extensive morbidity and mortality and are a major economic burden within disease-endemic countries. ${ }^{1,2)}$ Control of both these diseases, which in most countries are primarily transmitted in and around the home, is difficult. Malaria and dengue are effectively managed through a combination of vector control, drugs and management of clinical illness. Although drug and vaccine development for malaria and dengue have recently received much attention, ${ }^{3)}$ there have been few initiatives on vector control.

Malaria is the most important parasitic disease of humans. Over 3 billion people live in malarious areas and the disease causes between 1 to 3 million deaths per year ${ }^{4,5)}$ with morbidity reaching 515 million cases. This disease is resurging and both increased drug resistance of the parasite and increased insecticide resistance of the Anopheles mosquito vectors are contributing to this. Malaria vector control relies on the use of an effective insecticide, most commonly through indoor resid-

\footnotetext{
* To whom correspondence should be addressed.

E-mail: hemingway@liv.ac.uk

Published online March 20, 2007

(C) Pesticide Science Society of Japan
}

ual spraying (IRS) or community-based deployment of insecticide impregnated bednets (ITN). There are numerous cases of insecticide resistance reported for Anopheles species. ${ }^{6}$ )

Dengue is transmitted by Aedes mosquitoes. Up to 2.5 billion people live under threat of dengue, with 50 million new infections and 24,000 deaths reported annually. ${ }^{7)}$ Most Aedes control relies on the application of larvicides and insecticide space spays. ${ }^{8)}$ Aedes aegypti, the main dengue vector, often breeds in drinking water. There are 5 insecticides approved by the World Health Organization (W.H.O.) for application to drinking water. Since the 1970's the organophosphate, temephos has been the most widely used insecticide, but increased resistance and lack of community acceptance has reduced the efficacy of this insecticide. ${ }^{8,9)}$

Extensive exposure of insect vectors to insecticides eventually selects for insecticide resistance. An understanding of the basis of insecticide resistance and how to manage it, is needed if chemical control is to be used, either in isolation, or as part of an integrated pest management strategy. The economics of developing, safely testing and marketing insecticides means that few novel compounds are likely to be developed for the control of disease vectors. Hence good stewardship of available public health insecticides, through active monitoring and management of resistance levels in field populations of insects is essential. 
This paper reviews the current methods available to monitor insecticide resistance in mosquitoes and outlines the potential for using these methods to manage the development of insecticide resistance.

\section{Insecticides}

The first synthetic organochlorine insecticide to be commercialized, DDT (dichlorodiphenyltrichloroethane) is still used extensively for malaria control. DDT was central to the W.H.O. global malaria eradication campaign, $1955-1969 .{ }^{10)}$ While agricultural use of DDT has now ceased, due to environmental persistence and reduced efficacy against resistant insects, it still has a vital role in mosquito control as a cost-effective and safe insecticide for indoor residual spraying. More recently, pyrethroids have been widely used for malaria control. They are the only class of insecticides recommended by the W.H.O. for use on ITNs ${ }^{11)}$ and are also available for IRS. DDT and its analogues act on the sodium channels of the nerve membranes. Pyrethroids, such as permethrin and deltamethrin, and the pseudo-pyrethroid etofenprox, have a similar mode of action to that of DDT.

Phosphorothioate insecticides, such as temephos, malathion and pirimiphos methyl share the same mode of action as carbamates, such as bendiocarb, binding to acetylcholinesterase at the nerve junction. These insecticides are used extensively for IRS, or in the case of temephos as a larvicide. While there are a number of larvicides available from different chemical classes there are no other main stream chemical classes recommended for use by IRS.

\section{Insecticide Resistance}

Insecticide resistance in mosquitoes threatens our long term ability to control disease vectors. Resistance is defined by W.H.O. as "the development of an ability or strain of some organisms to tolerate doses of a toxicant that would prove lethal to a majority of individuals in a normal population of the same species." ${ }^{12)}$ Resistance is a genetically inherited characteristic which increases in the vector population as a direct result of the selective effects of the insecticide. ${ }^{13)}$ Genetic and phenotypic resistance arises in individuals as a result of mutation or gene duplication that results in the modification of a normal physiological, morphological or behavioral aspect of the mosquito phenotype. Such phenotypic changes typically enhance the process of detoxification of the insecticide or reduce the sensitivity of the nervous system to the insecticide. Insecticide resistant individuals have a higher probability of surviving over susceptible individuals when an insecticide is applied. As a result the resistance gene will increase in the population over time.

Insecticide resistance can be detected and investigated at many levels, from molecular characterization of genes conferring resistance and their biochemical products, to the role that gene products play in overcoming the toxic effects of insecticides. Figure 1 shows the mapping of insecticide resistance in
Southern Mozambique using a mixture of W.H.O. bioassays and biochemical assays.

\section{Mechanisms of Insecticide Resistance}

For detailed reviews of insecticide resistance mechanisms the reader is referred to a number of publications. ${ }^{12-15)}$ Although it is not the purpose of this paper to review resistance in detail a brief summary is given below.

A small group of enzyme families are involved in metabolic resistance. None of the enzymes are unique to resistant insects. Resistance can be due to a structural change in the enzyme molecule, that increases its ability to detoxify the insecticide, and/or an increase in the amount of the enzyme produced.

Cytochrome p450 monooxygenase (p450's) are a large group of oxidative enzymes with overlapping substrate specificities. Over 86 members of this large gene family are present in Drosophila melanogaster with the p450 family having undergone an expansion to 106 in Anopheles gambiae and further expansion again in $A$. aegypti. ${ }^{15,16)}$ Members of this enzyme family confer resistance primarily to pyrethroids and carbamates, and a lesser extent to organochlorines and organophosphates. They are also responsible for activating the phosphorothioate insecticides to their active oxon analogues.

Esterases are important in resistance to organophosphate insecticides and to a lesser extent pyrethroids. ${ }^{17)}$ These enzymes have been studied extensively in the mosquito Culex quinquefasciatus, where increased levels of one or more esterases, due to gene amplification, are responsible for resistance. ${ }^{17,18)}$ In several Culicine species the esterases act by sequestration of the insecticides. In contrast, in many Anophelines, a malathion carboxylesterase-type mechanism produces resistance through increased metabolism of the insecticide. $^{19,20)}$

Glutathione S-transferases (GST's) are primarily involved in DDT, pyrethroid and organophosphate resistance. Elevated GST levels have been studied in Anopheles gambiae and An. dirus, where resistance is primarily due to changes in the regulation of one or more GST families. ${ }^{21)}$

Alteration of the insecticides target site leads to insecticide site insensitivity. Alterations have been observed in neuronal enzymes and receptors, which are the target site of the majority of insecticides used in vector control.

Knockdown resistance $(k d r)$ occurs due to one or more mutations in the para-gated sodium channel gene. As this is the target site of DDT and pyrethroids, this mechanism produces cross-resistance to the two insecticide classes. In An. gambiae two mutations, Leu-Phe ${ }^{19)}$ and Leu-Ser, ${ }^{20)}$ have been identified at the same codon. Organophosphate and carbamate insecticides inhibit acetylcholinesterase (AChE). Many insect vectors have developed resistance through structural alterations of this target site. ${ }^{2,23)}$ These point mutations may act individually or in combination. 


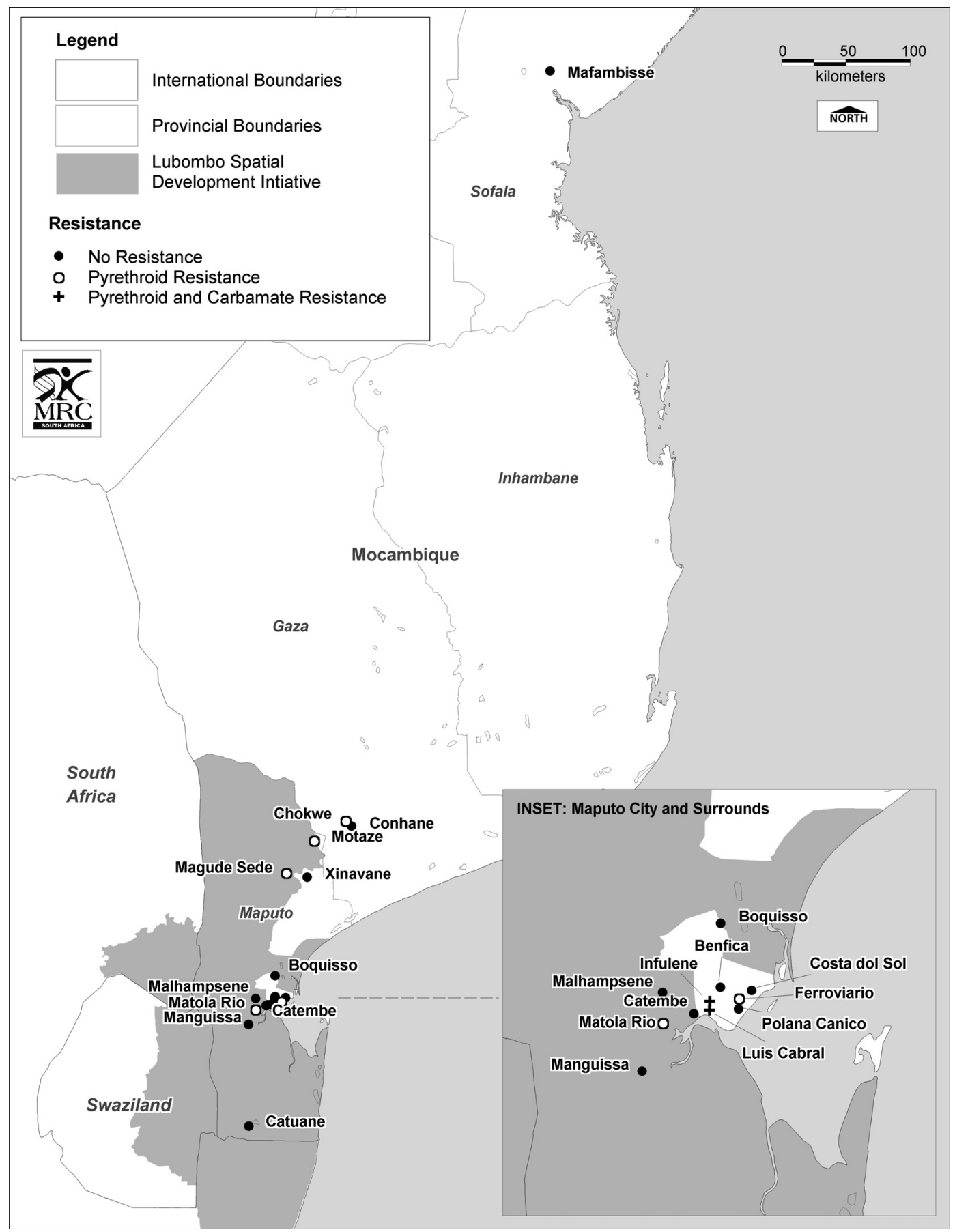

Fig. 1. Insecticide resistance at 18 localities in An. arabiensis in Mozambique.

\section{Quantifying Insecticide Resistance}

Insecticide resistance data needed to inform effective vector control policy has never been collected in a systematic manner. An attempt was made to rectify this problem for malaria, by the collation of published literature ${ }^{24)}$ and initiatives by the Multilateral Initiative on Malaria, which funded capacity building in South, West and East Africa for three short term insecticide resistance monitoring programs. However, a sus- tainable sentinel site system to monitor mosquito density and insecticide resistance still needs to be established in most disease endemic countries.

In order to plan effective resistance management simple assays to monitor and evaluate insecticide resistance and its underlying mechanisms are required. A number of biochemical and molecular assays have been developed, some of which can be carried out in the field. Applicable methods for resistance monitoring of field populations of insects are briefly 
summarized below.

\section{W.H.O. Diagnostic Assays ${ }^{25}$}

These are the most widely used assays in the field. Insects are exposed to filter papers impregnated with insecticide in a carrier oil formulation. The insecticide dosage is set at twice that which kills $100 \%$ of three day old non-blood fed adult females of known susceptible laboratory colonies for a range of Anopheline mosquitoes. While this is a robust dosage that susceptible individuals are unlikely to survive by chance, and W.H.O. guidelines suggest that resistance is only indicated if more that $5 \%$ of insects survive the exposure, reducing the risk of false positives, many heterozygous resistant individuals may be killed by the dose. Hence resistance levels may need to be very high before they are detected using this methodology. The discriminating dosages for Aedes aegypti are potentially more accurate, having been established for a single species rather than all Culicine mosquitoes.

A current list of recommended diagnostic doses for many insecticides for a number of arthropod disease vectors is published by W.H.O., and impregnated papers and test kits can be bought from a centralized distribution centre in Penang. ${ }^{26)}$ Most resistance reports for Anopheles in Africa and Aedes worldwide are based on the use of this methodology. ${ }^{6}$

These assays (with the potential exception of dieldrin bioassays) cannot be used to accurately monitor resistance gene frequencies in mosquito populations. They also give no indication of the underlying mechanisms of resistance, hence they have no predictive value for anticipating cross-resistance between insecticides.

\section{CDC Bottle Assays ${ }^{27)}$}

These are similar to the W.H.O. discriminating dose assays. Insecticide impregnated bottles are prepared by coating glass bottles with an acetone or alcohol based formulation. Insects are then exposed to the insecticides in the bottles. This assay has the advantage over the W.H.O. test kit that the rate of insecticide knock down is easier to score during the course of the exposure period. With rapid acting insecticides, such as pyrethroids this can be predictive of a $k d r$-type resistance mechanism within the population, although care should be taken not to over-interpret such data, as several effective metabolic resistance mechanisms also produce a reduced knock-down phenotype without any accompanying change in sensitivity at the sodium channel target site.

Bioassay data generated by either the CDC or W.H.O. method is a good indicator of the presence of resistance in mosquito populations, but they cannot be used to measure gene frequency accurately or suggest the epidemiological impact of resistance. Resistance gene frequencies in general will be higher than indicated by bioassay data alone. ${ }^{28,29)}$ Hence bioassays are not sufficiently sensitive to monitor low level resistance. A central theme of all resistance management modeling is that resistance needs to be detected at very low fre- quencies, hence methods that facilitate measuring the frequency of different mechanisms of resistance in field populations of mosquitoes are required. Methods currently available vary in their sophistication and ease of use.

\section{Biochemical Detection of Resistance}

A number of simple biochemical assays ${ }^{30)}$ are available to detect increased activity of three enzyme systems, esterases, ${ }^{31-33)} \mathrm{GST}^{34)}$ and $\mathrm{p} 450$ ' $^{35)}$ involved in insecticide metabolism. Many of these assays detect increased enzymatic activity against model substrates in resistant individuals.

One of the most common metabolic resistance mechanisms in Culicine mosquitoes involves gene amplification of one or more esterase that sequester organophosphates and slowly turn them over. Initial methods for elevated esterase detection were filter paper-based ${ }^{36}$, having the advantage of producing permanent records of results, but the method had the disadvantage that esterase, and hence resistance levels were not easily quantifiable. Later methods were microtitre plate based, allowing accurate quantification of esterase levels with access to a plate reader, although results could still be scored by eye as with the filter paper tests. Biochemical assays for the GSTs and p450's are less field applicable. The GST microtitre plate assay, although accurate, requires access to a plate reader able to measure absorbance at $340 \mathrm{~nm}$. There is no direct assay for p450 activity in individual insects. A modified haem assay allows a very crude estimation of the amount of $\mathrm{p} 450$ present in single insects, but results are difficult to interpret, detecting only very high levels of enzyme elevation.

Only one of the target sites, acetylcholinesterase (AChE) is amenable to development of a biochemical detection system. ${ }^{37)}$ A simple microtitre plate assay is available to measure AChE insensitivity using a carbamate or an oxon analogue of a phosphorothioate insecticide. In contrast to all the other metabolic microtitre plate assays, this assay is sufficiently accurate to measure resistance gene frequencies, allowing differentiation between homozygous and heterozygous resistant individuals.

\section{Molecular Methods for Resistance Detection}

Mutations in the insecticides target site lend themselves to detection through simple PCR assays which can readily be used in many field settings. Allele specific PCR assays have been developed for three major target sites, the GABA receptors, ${ }^{38,39)}$ the sodium channels $(k d r)^{40,41)}$ and AChE. The challenge is to adapt these assays for high throughput field application, as they have the advantage of detecting heterozygous resistant individuals that may be missed by other assays.

All enzymes involved in detoxifying insecticides belong to large enzyme families, members of which have varying substrate specificities. In many cases of insecticide resistance which have a metabolic basis, the exact molecular mechanism to resistance is unknown, hence allele specific assays are not yet available. However, recent advances in genomics has al- 
lowed a much more rapid identification of genes that are up or down regulated in insecticide resistant insects using microarray technology. ${ }^{42)}$ The detoxification microarray chip, developed for An. gambiae, contains all potential insecticide resistance genes. Population screening using this chip has allowed the rapid identification of genes that are up or down regulated in resistant compared to susceptible insects. These differentially regulated genes are being expressed to directly assess their ability to metabolize or bind insecticide. The availability of this technique has reduced the time required to fully document the resistance genes within a population from years to months. Positional cloning approaches have then confirmed the co-location of these up-regulated genes with the physical location of the insecticide resistance quantitative trait locus. Once resistance genes have been identified they can then be screened for allele specific single nucleotide polymorphisms (SNPs) that segregate with the resistance phenotype and the SNPs will then form the basis of a simple PCR type assay that can be used routinely in field populations. Combining the high technology approach of microarrays with routine population monitoring with simple PCR technology will afford a better way of accurately monitoring the frequency of known resistance genes in field populations of mosquitoes. ${ }^{43)}$ This technology also has the added advantage over bioassays that it can be undertaken on dead mosquitoes. The biochemical assays, while they can be undertaken on dead mosquitoes, require that the mosquitoes have not had immediate prior exposure to insecticides and that the mosquitoes have been preserved by freezing after death. The first prototype field kits for resistance detection at the molecular level should be available for testing towards the end of 2007.

The detoxification chip can also be used to screen for resistance genes in other Anopheles species. Screens have already been successfully undertaken on An. stephensi and work is underway on pyrethroid resistant An. funestus. A similar detoxification chip has been developed for $A$. aegypti and currently a large number of insecticide resistant strains are being screen against this chip to identify the major metabolic resistance genes in this species, which is the major dengue vector.

\section{Insecticide Resistance Management}

Resistance threatens the long term ability of humans to control diseases particularly as resistance is occurring at a faster pace than new insecticides are being developed. The historical response of waiting until an epidemiological end point for disease to assess if vector control has failed is no longer sustainable, insecticide resistance management is essential to conserve scarce public health insecticides.

With only four classes of insecticides recommended for the control of adult mosquitoes it is vital that effective resistance management strategies are employed. The aim is to prevent or delay the onset of resistance in populations exposed to an insecticide or develop management programs that cause exist- ing resistance in populations to decline.

The impact of resistance on disease control can be dramatic as in the case of southern Africa where resistance to the malaria vector An. funestus to pyrethroids ${ }^{44)}$ was correlated to a dramatic increase in malaria incidence in the region.

Resistance management strategies take advantage of the adverse fitness costs of resistance genes, to the insects carrying them, in the absence of insecticide selection pressure. Random genetic events generate mutant alleles some of which confer insecticide resistance. Alleles with strong pleiotropic effects are generally selected against in the absence of selection pressure. When insecticide selection pressure is applied, the frequency of resistant alleles increases. The dominance status of the trait is important as this can affect the outcome of resistance management strategies.

Computer models have identified some key factors affecting the evolution of resistance. ${ }^{44-47)}$ These models provide a simple means of predicting the efficacy of different management strategies. However, models often lack critical information such as population size, migration rates, selection intensity and the fitness of alleles. This lack of information undermines the models. Several models have been tested under laboratory conditions but rarely in field conditions. Utilizing the tools currently available for monitoring insecticide resistance an ongoing trial in southern Mexico compares changes in frequencies of resistant alleles under mosaic and rotational resistant management strategies to single insecticide use. ${ }^{48}$ )

Using a single insecticide assumes that the initial frequency of the resistant allele is low and the vast majority exist as heterozygotes. Applying an insecticide sufficiently high enough to kill all heterozygotes has been advocated. The frequency of homozygote resistant mosquitoes is assumed to be so low they would be overwhelmed and mate with homozygous susceptible immigrant mosquitoes. For this approach to succeed all heterozygous individuals must receive the appropriate lethal dose. This is difficult to achieve under field conditions and is costly with environmental implications.

An alternative is to utilise two or more insecticides, the aim being that resistance will evolve more slowly to both insecticides. Mixtures of insecticides, require the expected frequency of resistant alleles at two different genetic loci to be low and that individual mosquitoes carrying both alleles are rare. Using two or more insecticides in a spatial pattern assumes that individual mosquitoes will be exposed to more than one insecticide with similar results to insecticide mixtures.

Temporal rotation where insecticides are applied in an alternating sequence is also based on the assumption that an individual mosquito does not carry two resistant alleles. Should the frequency of an allele increase in a population under selection by an insecticide, they will be killed when the switch is made to the next insecticide. It is assumed that the resistant gene will have a selective disadvantage during the absence of selection pressure. If this assumption does not hold true, rota- 


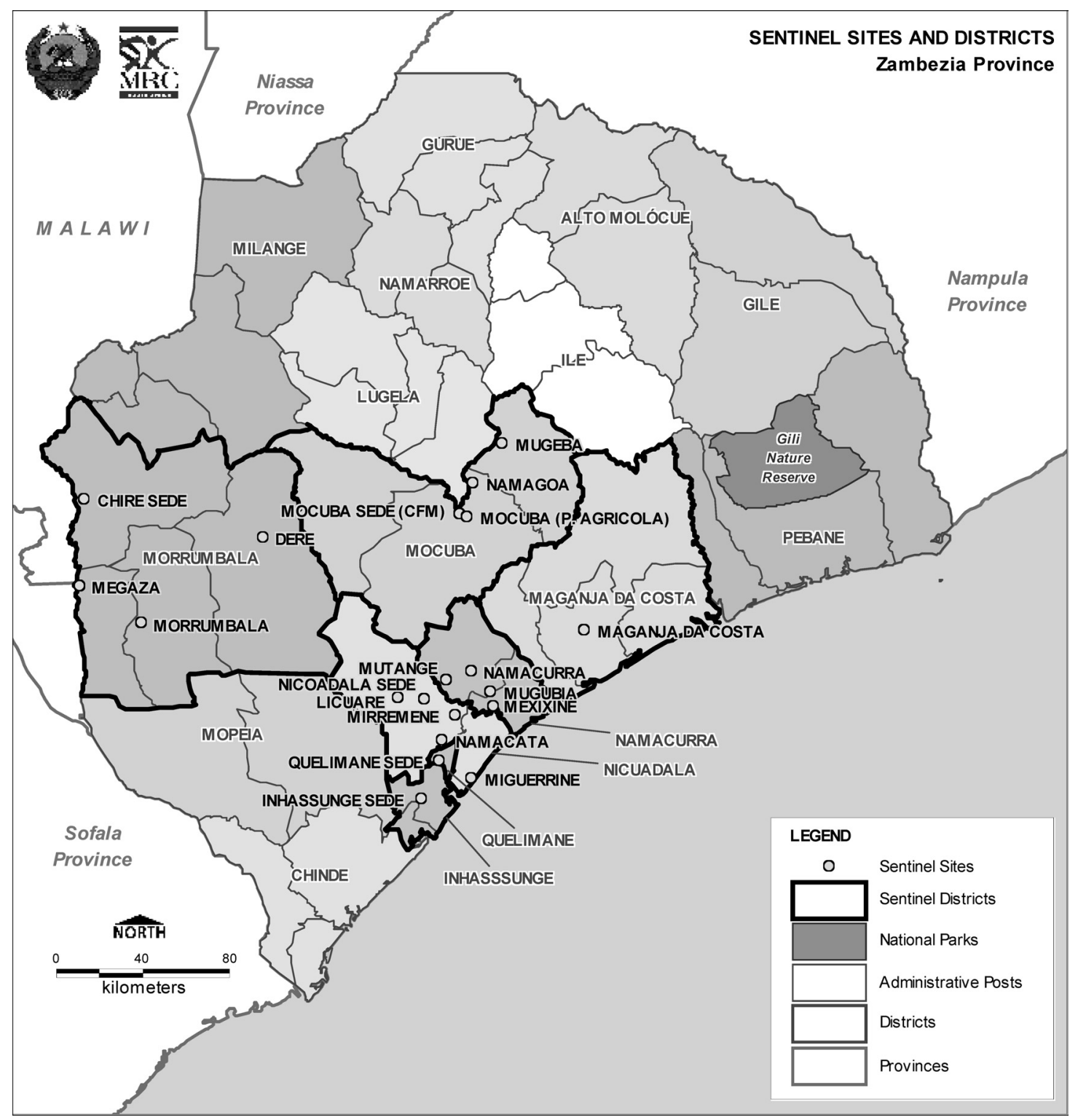

Fig. 2. The density of sentinel sites required to support a malaria decision support system incorporating entomological data.

tion of insecticides will not prevent the accumulation of resistant alleles.

There are few long term field studies on the resistance gene frequencies to asses the fitness costs in natural populations. The common prediction of models is that resistance will reduce without selection pressure. This has been demonstrated in the mosquito Culex pipiens ${ }^{49)}$ and sheep blow fly Lucilia cuprina. $^{50)}$

\section{Decision Support Systems}

The potential for resistance to develop in Anopheles and Aedes vectors has been apparent since the 1950 's ${ }^{51)}$ but the problem has been poorly documented. As a first step to improve this system a database of published insecticide resistance available for Anopheles in Africa was collated. ${ }^{52)}$ This demonstrated the limited amount of information available to the malaria control community. The W.H.O. led African Network for Vector Research (ANVR) aimed to fill in some of these gaps. This information has not yet made it into main stream literature. To date no comprehensive map of resistance has been established for Aedes.

Currently insecticide resistance in vectors is not monitored directly and is only detected once an operationally significant increase in disease transmission occurs. A decision support system (DSS) utilizing geographical information system technology has recently been established and used operationally in Mozambique, but does not as yet incorporated spatial or temporal data on vector abundance or insecticide resistance. Figure 2 shows the spatial scale on which sentinel sites need to be established for such a system. The incorporation of insecti- 
cide resistance data into decision support systems for malaria and dengue control will allow for evidence-based decisions on control.

The benefits of insecticide evidence-based vector control has been established in SriLanka, ${ }^{53)}$ Mexico $^{48)}$ and Mozambique. $^{28,54)}$ The Innovative Vector Control Consortium $(\text { IVCC })^{55)}$ will develop the new tools to quantify insecticide resistance in the field and the DSS required to make informed insecticide choice. The IVCC is currently further developing the available malaria DSS to incorporate more entomological data allowing more effective control of vectors through prompt timely and focused application of insecticides which will delay insecticide resistance.

\section{References}

1) J. Sachs and P. Malaney: Nature 415, 680-685 (2002).

2) A. Boutayeb: Trans. R Soc. Trop. Med. Hyg. 100, 191-199 (2006).

3) L.J. da Silva and R. Richtmann: J. Pediatr. (Rio J) 82, S115S124 (2006).

4) C. A. Guerra, R. W. Snow and S. I. Hay: Adv. Parasitol. 62,157-179 (2006).

5) R. W. Snow, C. A. Guerra, A. M. Noor, H. Y. Myint and S. I. Hay: Nature 434, 214-217 (2005).

6) M. Coleman, B. Sharp, I. Seocharan and J. Hemingway: J. Med. Entomol. 43, 663-668 (2006).

7) B. A. da Fonseca and S. N. Fonseca: Curr. Opin. Pediatr. 14, 67-71 (2002).

8) J. B. Lima, M. P. Da-Cunha, R. C. Da Silva, A. K. Galardo, S. S. Soares, I. A. Braga, R. P. Ramos and D. Valle: Am. J. Trop. Med. Hyg. 68, 329-333 (2003).

9) C. Brengues, N. J. Hawkes, F. Chandre, L. McCarroll, S. Duchon, P. Guillet, S. Manguin, J. C. Morgan and J. Hemingway: Med. Vet. Entomol. 17, 87-94 (2003).

10) P. I. Trigg and A. V. Kondrachine: Bull. World Health Organ. 76, 11-16 (1998).

11)) W.H.O.: World Health Organ. Tech. Rep. Ser. 443, 1-279 (1970).

12) J. Hemingway and H. Ranson: Annu. Rev. Entomol. 45, 371-391 (2000).

13) A. W. Brown: J. Am. Mosq. Control Assoc. 2, 123-140 (1986).

14) S. L. R. Casimiro: University of Natal, South Africa. Thesis/Dissertation

15) H. Ranson, C. Claudianos, F. Ortelli, C. Abgrall, J. Hemingway, M. V. Sharakhova, M. F. Unger, F. H. Collins and R. Feyereisen: Science 298, 179-181 (2002).

16) H. Ranson, D. Nikou, M. Hutchinson, X. Wang, C. W. Roth, J. Hemingway and F. H. Collins: Insect Mol. Biol. 11, 409-418 (2002).

17) A. Vaughan, M. Rodriguez and J. Hemingway: Biochem. J. 305, 651-658 (1995).

18) C. Mouches, Y. Pauplin, M. Agarwal, L. Lemieux, M. Herzog, M. Abadon, V. Beyssat-Arnaouty, O. Hyrien, de S. V. and G. P. Georghiou: Proc. Natl. Acad. Sci. USA 87, 2574-2578 (1990).

19) D. Martinez-Torres, F. Chandre, M. S. Williamson, F. Darriet, J. B. Berge, A. L. Devonshire, P. Guillet, N. Pasteur and D. Pau- ron: Insect Mol. Biol. 7, 179-184 (1998).

20) H. Ranson, B. Jensen, J. M. Vulule, X. Wang, J. Hemingway and F. H. Collins: Insect Mol. Biol. 9, 491-497 (2000).

21) A. A. Enayati, H. Ranson and J. Hemingway: Insect Mol. Biol. 14, 3-8 (2005).

22) M. Weill, C. Malcolm, F. Chandre, K. Mogensen, A. Berthomieu, M. Marquine and M. Raymond: Insect Mol. Biol. 13, 1-7 (2004).

23) R. N'Guessan, F. Darriet, P. Guillet, P. Carnevale, M. TraoreLamizana, V. Corbel, A. A.Koffi and F. Chandre: Med. Vet. Entomol. 17, 19-25 (2003).

24) M. Coleman, B. Sharp, I. Seocharan and J. Hemingway: J. Med. Entomol. 43, 663-668 (2006).

25) W.H.O.: WHO/VBC/81.806, 1-7. 1981. Geneva, WHO.

26) W.H.O.: WHO/CDS/CPC/MAL/98.12. 1998. Geneva.

27) W. G Brogdon and J. C. McAllister: Emerg. Infect. Dis. 4, 605-613 (1998)

28) S. Casimiro, M. Coleman, J. Hemingway and B. Sharp: J. Med. Entomol. 43, 276-282 (2006).

29) S. Casimiro, M. Coleman, P. Mohloai, J. Hemingway and B. Sharp: J. Med. Entomol. 43, 267-275 (2006).

30) W.H.O.: WHO/CDS/CPC/MAL/98.6. 2000.

31) W. G. Brogdon: Comp. Biochem. Physiol. C 90, 145-150 (1988).

32) O. Dary, G. P. Georghiou, E. Parsons and N. Pasteur: J. Econ. Entomol. 83, 2187-2192 (1990).

33) C. Brengues, N. J. Hawkes, F. Chandre, L. McCarroll, S. Duchon, P. Guillet, S. Manguin, J. C. Morgan and J. Hemingway: Med. Vet. Entomol. 17, 87-94 (2003).

34) W. G. Brogdon and A. M. Barber: Comp. Biochem. Physiol. B 96, 339-342 (1990).

35) W. G. Brogdon, J. C. McAllister and J. Vulule: J. Am. Mosq. Control Assoc. 13, 233-237 (1997).

36) O. Dary, G. P. Georghiou, E. Parsons and N. Pasteur: J. Econ. Entomol. 84, 28-33 (1991).

37) J. Hemingway and C. Smith: Bull. Entomol. Res. 76, 559-565 (1986).

38) W. Du, T. S. Awolola, P. Howell, L. L. Koekemoer, B. D. Brooke, M. Q. Benedict, M. Coetzee and L. Zheng: Insect Mol. Biol. 14, 179-183 (2005).

39) R. H. ffrench-Constant, J. C. Steichen and F. Shotkoski: Med. Vet. Entomol. 8, 99-100 (1994).

40) D. Martinez-Torres, F. Chandre, M. S. Williamson, F. Darriet, J. B. Berge, A. L. Devonshire, P. Guillet, N. Pasteur and D. Pauron: Insect Mol. Biol. 7, 179-184 (1998).

41) A. Lynd, H. Ranson, P. J. McCall, N. P. Randle, W. C. Black, E. D. Walker and M. J. Donnelly: Malar. J. 4, 16 (2005).

42) J. P. David, C. Strode, J. Vontas, D. Nikou, A. Vaughan, P. M. Pignatelli, C. Louis, J. Hemingway and H. Ranson: Proc. Natl. Acad. Sci. USA 102, 4080-4084 (2005).

43) K. Hargreaves, L. L. Koekemoer, B. D. Brooke, R. H. Hunt, J. Mthembu and M. Coetzee: Med. Vet. Entomol. 14, 181-189 (2000).

44) C. Boete and J. C. Koella: Malar. J. 1 :3, 3 (2002).

45) M. J. Livingston, G. A. Carlson and P. L. Fackler: J. Econ. Entomol. 95, 1008-1017 (2002).

46) M. Raymond, D. G. Heckel and J. G. Scott: Genetics 123, 
543-551 (1989).

47) G. S. Mani: Genetics 109, 761-783 (1985).

48) J. Hemingway, R. P. Penilla, A. D. Rodriguez, B. M. James, W. Edge, H. Rogers and M. H. Rodrigez: Pestic. Sci. 51, 375-382 (1997).

49) A. Yebakima, M. Marquine, J. Rosine, M. M. Yp-Tcha and N. Pasteur: J. Med. Entomol., 41, 718-725 (2004).

50) A. C. Kotze and N. Sales: J. Econ. Entomol. 94, 1243-1248 (2001).

51) W.H.O. Malaria Section: Bull. World Health Organ. 16, 874 (1957).
52) M. Coleman, B. Sharp, I. Seocharan and J. Hemingway: J. Med. Entomol. 43, 663-668 (2006).

53) L. A. kelly-Hope, M. Yasuno, M. B. Wickramasinghe, M. D. Perera, S. H. Karunaratne, W. P. Fernando, R. R. Abeyasinghe, R. R. Siyambalagoda, P. R. Herath, G. N. Galappaththy and J. Hemingway: Trans. R. Soc. Trop. Med. Hyg. 99, 751-761 (2005).

54) S. Casimiro, M. Coleman, P. Moholai, J. Hemingway and B. Sharp: J. Med. Entomol. 43, 267-275 (2006).

55) J. Hemingway, B. J. Beaty, M. Rowland, T. W. Scott and B. L. Sharp: Trends Parasitol. 22, 308-312 (2006). 